\title{
Perceived Technology Clusters and Ownership of Related Technologies: The Case of Consumer Electronics
}

\author{
Frank J. Van Rijnsoever and Carolina Castaldi \\ Faculty of Geosciences, Innovation Studies Utrecht, Utrecht University, P.O. Box 80115, 3508 TC Utrecht, \\ The Netherlands. E-mail: \{f.vanrijnsoever, C.Castaldi\}@geo.uu.nl
}

\begin{abstract}
We contribute to the understanding of how technologies may be perceived to be part of technology clusters. The value added of the paper is both at a theoretical and an empirical level. We add to the theoretical understanding of technology clusters by distinguishing between clusters in perceptions and clusters in ownership, and by proposing a mechanism to explain the existence of clusters. Our empirical analysis combines qualitative and quantitative methods to investigate clusters of consumer electronics for a sample of Dutch consumers. We find that perceived clusters in consumer electronics are mostly determined by functional linkages, and that perceived technology clusters are good predictors of ownership clusters, but only for less widely diffused products.
\end{abstract}

\section{Introduction}

In his famous book on the diffusion of innovations, Rogers (2003, p. 249) states that "innovations are often not viewed singularly by individuals, but they may be perceived as an interrelated bundle of new ideas. The adoption of one idea may trigger the adoption of others." This intuition has been taken up by a few researchers that have further developed the seminal idea of perceived related technologies to the concept of technology clusters, and have tested it in practice. ${ }^{1}$ The word technology has been used to refer to technology-based innovations, and has most often been applied in the field of information technologies. While the claim in Rogers implicitly assumes that an innovation entails a new idea, technology clusters specifically refer to new ideas embodied in actual products.

The motivation for an interest in technology clusters has been spurred by the empirical evidence that such clusters

Received June 6, 2008; revised September 4, 2008; accepted September 4, 2008

\footnotetext{
${ }^{1}$ Some authors prefer the term innovation clusters (see LaRose \& Hoag, 1996 and Neuendorf, Atkin, \& Jeffres, 1998). Both terms are in fact also used in the literature on industrial clusters for clusters of technology/innovationoriented firms.
}

(C) 2008 ASIS\&T $\bullet$ Published online 30 October 2008 in Wiley InterScience (www.interscience.wiley.com). DOI: 10.1002/asi.20975 can be significant predictors of the adoption of innovations (see for instance Busselle Reagan, Pinkleton, and Jackson, 1999; Lin, 1998; and Vishwanath \& Goldhaber, 2003). They have, for example, been defined by shared infrastructures (LaRose \& Atkin, 1992; Perse \& Courtright, 1993) or by brand (Warlop, Ratneshwar, \& Van Osselaer, 2005). Clusters have also been determined in relation to the lifestyle of the adopter (Ettema, 1984), to some emotional attachment (Kwortnik \& Ross, 2007), or to the fulfilment of communication needs (Cowles, 1989; Perse \& Courtright, 1993).

In this paper we propose that the literature on technology clusters can take further steps in two main directions. First, as discussed in Vishwanath and Chen (2006), technology clusters have been used and defined in ad hoc ways depending on the focus of the study. The definition proposed by Rogers (2003) implies that there is a relationship between two different types of clusters, namely that a perceived relationship between products (perceived clustering) is predictive of the combined ownership of these products (ownership clustering).

Perceived relationships among products are the focus of product categorization literature (see, e.g., Nedungadi, Chattopadhyay, \& Muthukrishnan, 2001; Rosa \& Porac, 2002), while the combined ownership of technologies is discussed in the technology adoption literature (Leung \& Wei, 1999). Most studies consider technology clusters as exogenous, and imply that clusters reflect past behavior. They do not aspire to formulate a theoretical mechanism that explains their existence. ${ }^{2}$ Mechanisms on how clusters come to exist can be formulated both for perceived technology clusters and for the combined ownership of technologies. The theoretical mechanism behind both perceived and ownership clusters is bound to depend on the specific technologies considered. We propose a theoretical mechanism for both types of clusters and we relate them by testing whether perceived clusters are a good predictor of actual ownership. Continuing the line of

\footnotetext{
${ }^{2}$ The studies by LaRose and Atkin (1992) and Vishwanath and Chen (2006) are the only two exceptions that we are aware of.
} 


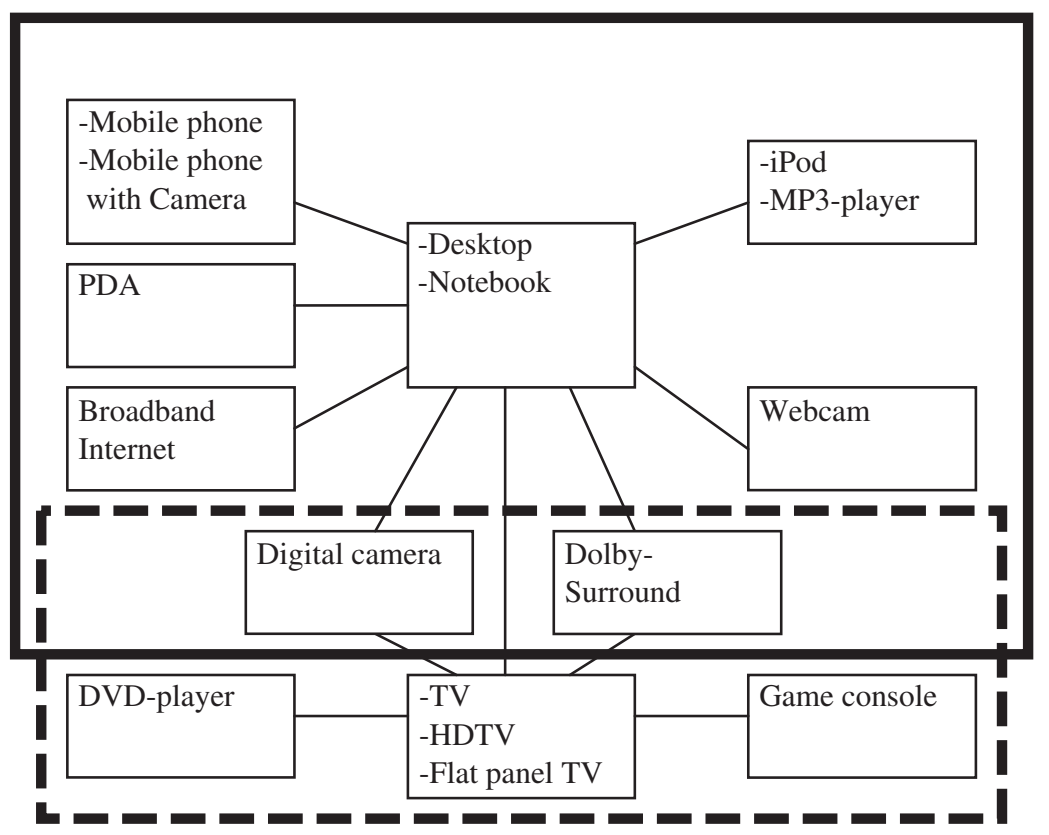

FIG. 1. The 16 technologies and their shared infrastructure. PDA (Personal Digital Assistant) HDTV (High Definition TV, iPod, Flat panel TV, Game console, Webcam, MP3 player, Notebook (or laptop), Dolby-surround, Mobile phone with camera, Digital camera, Broadband Internet, Desktop, DVD player, Mobile phone, TV.

most previous studies on this topic, we apply our theory to information-related consumer electronic products.

Second, Vishwanath and Chen (2006) have suggested that different types of adopters may perceive technology clusters differently. They find that early adopters perceive technologies to be related through functional interdependencies and a shared infrastructure, while nonadopters relate technologies based upon their functional merits. Their contribution is a first step towards a better understanding of the individual characteristics of adopters that shape technology clusters. In this paper we analyze the role of consumers' prior adoption in determining the likelihood of linking two technologies together.

In the next section we develop a theoretical framework for technology clusters in consumer electronics. Next, we test our hypotheses on a sample of Dutch consumers using a combination of qualitative and quantitative research methods. In the conclusion we discuss the implications of our results for the literature on technology clusters and suggest some implications for consumer behavior scholars as well.

\section{Theoretical Framework}

\section{Technology Clusters in Consumer Electronics}

Following Rogers (2003), a technological innovation ${ }^{3}$ can be defined as a technology/product that is perceived to be new by an individual. This innovation can be viewed as being stand-alone or as being part of a perceived larger whole,

\footnotetext{
${ }^{3}$ In what follows we shall simply refer to innovations and use the term interchangeably with new products and new technologies.
}

a technology cluster (LaRose \& Hoag, 1996; Rogers, 2003; Vishwanath \& Chen, 2006).

Figure 1 graphically displays 16 different technologies ${ }^{4}$ that are considered in this study. The underlying infrastructure is also depicted. The lines that connect the technologies display possible physical connections, like cables or Bluetooth, between them. The hubs in the infrastructure can be considered "base technologies": they are stand-alone equipment to which other devices can be linked so that the performance of either of the two devices increases. For consumer electronics two base technologies can be identified, the personal computer (PC) and the television (TV). ${ }^{5,6}$ The PC is represented by the desktop and the notebook, which have similar functions and can be considered to a great extent as functional substitutes. Together with ordinary television, we consider two functional substitutes, high definition television (HDTV) and flat panel television (FPTV). All other technologies are considered "peripheral." Peripheral technologies are functional complements of the respective base technologies. This complementarity entails a strong linkage between peripheral and base technologies since the proper

\footnotetext{
${ }^{4}$ The products chosen cover a wide range in terms of diffusion (from TV to PDA). We sought for a relatively complete list of consumer electronics while at the same time limiting the number of products to 16 in order to keep the response rate of our questionnaire high.

${ }^{5}$ It can be argued whether the TV is actually a base technology, because it needs an input signal to function. Such a signal could also be viewed as a separate technology. In this study it is assumed that all TVs owned by the respondents have an input signal in the form of a cable, antenna, or satellite. This assumption is reasonable for a country like the Netherlands, but it may be reconsidered for a country with a weaker infrastructure.

${ }^{6} \mathrm{~A}$ cluster around the TV has previously been identified by Perse and Courtright (1993)
} 
functioning of peripheral technologies is contingent upon the ownership of the corresponding base technology.

\section{Perceived Clusters in Consumer Electronics}

According to Rosa and Porac (2002) the categorization of products by individuals depends on how the products are experienced, which in turn largely depends on contextual factors. Yeh and Barsalou (2006) propose a general classification of properties on which cognitive categories for tangible objects can be based. Their classification can be used to understand which properties define categories of products in consumer electronics. They distinguish among categories based on entity properties (e.g., small phones, thin TVs), situational properties that describe the physical setting or event with which the product is associated (e.g., conversing, hearing ring tone and beeps for the mobile phone), taxonomic properties (neighboring concepts in a cognitive taxonomy like music devices), and introspective properties, which describe agents' subjective perspective on the target object (e.g., annoying devices, convenient products). Products sharing common properties fall into the same perceived category.

Following the representation in Figure 1, we consider categories of products based on linkages defined by "basic functions," an example of categorization based on taxonomic properties. The basic function of a product is defined as the primary function for which the product is purchased. For example, the basic function of a television is to display video transmissions from several sources (e.g., DVD player, Cable-TV, Satellite, Game console, etc.) We define four different categories of linkages and corresponding indicators of "infrastructural distance" between technologies:

- Overlapping functions (OF): technologies perform the same basic function, in other words, they are largely functional substitutes. For example, a notebook basically does the same as a desktop computer. The infrastructural distance between the technologies is zero.

- Functional interdependencies (FI): the technologies are directly connected to each other and the performance of either technology depends on this connection; they are functional complements. For example, broadband Internet does not function without a computer. The infrastructural distance between the technologies is one.

- Shared base technology (SBT): the technologies are connected with each other through a base technology. A webcam for example is connected to the Internet through a computer. The infrastructural distance between the technologies is two.

- Unknown (Unk): This category entails all other linkages that cannot be related to a functional linkage, but relate instead to entity, situational, or introspective properties (such as lifestyle- or brand-related properties). The distance between the technologies is three or greater. The word Unknown thus means that the type of link is unknown when clusters are viewed from a shared-infrastructure perspective.

Appendix A shows how we classified each of the 120 possible links among the 16 technologies. ${ }^{7}$ The classification

\footnotetext{
${ }^{7}\left(16^{2}-16\right) / 2=120$
}

stems directly from Figure 1 and from the four categories defined above: functional substitutes are classified as OL, and functional complements as FI.

Given the existence of clear base technologies in the case of consumer electronics, we suggest that linkages based on infrastructural distance will be significant predictors for perceived technology clusters. Specifically, we assume that a lower infrastructural distance is associated with a higher likelihood for perceiving technologies as part of the same cluster. This implies two main claims. First, products with overlapping functions (substitutes) are most likely to be perceived as being part of the same cluster. Second, factors different from functional linkages, falling in our "Unknown" category, matter the least for predicting clusters in consumer electronics. Our first hypothesis is then:

Hypothesis 1: The larger the infrastructural distance between two technologies, the smaller the likelihood of perceived linking between them.

\section{Prior Adoption and Perceived Technology Clusters}

As discussed in the introduction, Viswanath and Chen (2006) found that adopters and non-adopters perceive relationships among technologies differently. Early adopters relate technologies based on their functional interdependence, while later adopters focus more on overlapping functions. These findings are in line with the Consumer Learning by Analogy model (CLA; Gregan-Paxton \& John, 1997). The theory considers "experts" as individuals with a larger prior knowledge and claims that experts perceive technologies to be related based on actual relationships or "links," like a shared infrastructure. On the other hand, nonexperts perceive technologies to be related based on single attributes (like having overlapping functions). This also implies that nonexperts use more entity properties and introspective properties than experts. Furthermore, Moreau, Markman, and Lehmann (2001) found that categorization for new products depends on external cues (situational properties and entity properties, but certainly not taxonomic concepts), because there is no existing knowledge base to fit them in. The level of prior adoption determines the extent to which an individual makes use of taxonomic concepts in associating products. In assessing technology clusters the level of expertise and thus the level of prior adoption can play an important moderating role in the type of link used to relate technologies. We propose the following hypothesis:

Hypothesis 2: Individuals with a high level of prior adoption of the products are more likely to perceive links based on functional interdependence, while individuals with a low level of prior adoption are more likely to perceive links based on overlapping functions.

\section{Perceived Clusters and Actual Ownership Clusters}

The relationship between perceived linking of technologies and actual ownership forms the basis of Rogers' (2003) 
argument on technology clusters. His starting assumption is that perceived clustering can enhance the likelihood of adoption. Logically, consumers will only purchase technologies that they can actually put to use. If one does not posses or has no access to a television, it is of little value to purchase a DVD player (for personal use). This means that ownership patterns are expected to follow the patterns laid out by the shared infrastructure. In the case of functional interdependencies clustering does favor adoption, but this is most likely when the performance of the base technology gets enhanced by the peripheral technology. If two different products have the same basic function, a consumer does not aspire to own both products. An example is the case of the iPod and an ordinary MP3 player. Since both play digital media, there is little reason for an individual consumer to own both products, except for a replacement purchase or because there are additional functions that one of the two devices does not have, but that are perceived to be important by particular consumers. Finally, the likelihood of adoption as a result of clustering decreases if there is only a shared base technology, and the peripheral technology has no added value from the other technologies.

Based on the above considerations, we expect the type of link to explain the difference between perceived clusters and observed ownership patterns. From this it follows that

Hypothesis 3: The relationship between perceived technological clusters and actual technology ownership is moderated by the type of link in such a manner that links based on functional interdependencies and a shared base technology have a higher likelihood of being found in ownership clusters than links based on overlapping functions.

We expect no effect of prior adoption in actual ownership patterns, because we view prior adoption as the combined ownership of technologies; this means that prior adoption is incorporated in our dependent variable.

We add a last but very important control factor to our model. In modelling perceived relationships we are only dependent on the preferences of the respondents. This is not the case for patterns in ownership; here diffusion of the technology through the population also plays a significant role. In testing our hypotheses we would like to test whether the actual patterns in ownership depart significantly from what we would expect to find on the basis of chance. However, in the case of two widely diffused technologies (the added percentages of ownership of both technologies is larger than 100\%) links will be formed not only by chance, but also because it is certain that both technologies are owned by some consumers. The amount of diffusion thus heavily influences our results. In our methodology we discuss how we deal with this issue.

\section{Empirical Studies}

As concerns our empirical analysis, we will combine the results of oral interviews with survey data. Most contributions in this field come from survey data (e.g., LaRose \&
Atkin,1992; LaRose \& Hoag, 1996; Leung \& Wei, 1999; Vishwanath \& Goldhaber, 2003). A notable exception is the paper by Vishwanath and Chen (2006), who take an original approach by using multidimensional scaling techniques.

To test our hypotheses two studies were conducted:

- A study with semistructured interviews combining a qualitative analysis to investigate how consumers perceive technology clusters and a statistical analysis to test Hypotheses 1 and 2 .

- A quantitative study to find out which technologies are actually owned in combination with each other and to compare them with the perceived clusters. This study seeks to confirm Hypothesis 3.

\section{Study 1: A Study Into Perceived Clusters}

Methods.

Sample and data collection. A group of 21 university students in a research methodology course conducted a series of 47 interviews with a sample of consumers. Although the sample size is too small to form an adequate representation of the population, quota by age and sex were used to ensure a broad sample. ${ }^{8}$ Respondents varied in age between 16 and 86 years old, with a mean of 48.55 . The sample consisted of 23 men and 24 women. All interviews were held in the respondents' own houses. The interviews were audio recorded and written out literally afterwards. At the beginning of the interview, the interviewer laid out in front of the respondent a series of 16 cards with the names and pictures of the 16 previously mentioned technologies shown in Figure 1. The cards were laid out in a predetermined format of two horizontal rows consisting of 8 cards each. The interviewer asked the respondents whether they would group the cards into, for them, logical clusters. To prevent influence through external cues (Moreau et al., 2001), no hint for the manner of clustering (such as hints for a category structure; Nedungadi et al., 2001) was given prior to this question. Respondents were told that if they required a technology to form a cluster more than once, they could receive a spare card. After the respondent had finished laying out the combinations, the interviewer wrote these down. Next the interviewer asked, for each cluster, why the respondents had made this particular combination of technologies. After giving their reasons, the respondents were asked which of the technologies they actually owned.

Analysis. For each respondent all cluster data was put into a $16 \times 16$ matrix, where the rows and columns stand for the 16 technologies; there were 120 different possible combinations. Each time two products were placed in the same group the value of the combination became 1 ; this represented a perceived link. If two products were not in the same group, then the combination received the value 0 .

\footnotetext{
${ }^{8}$ The study by Vishwanath and Chen (2006) only addressed young consumers. Our sample includes consumers of all ages and thus deals with one of the further tests indicated by the two authors in their conclusions.
} 
All relations were coded in a manner based on our theory (see Appendix A): 1 if the products have overlapping functions, 2 if the products are functionally interdependent, 3 if the products share the same base technology, and 4 for unknown.

There are two levels at which we can analyze our data: we can analyze the aggregated matrices of the entire sample, or we can analyze the matrix of each respondent separately. This implies a two-level model (Snijders \& Bosker, 1999), with the possible combinations at the macrolevel and each respondent at the microlevel. In this case we prefer a twolevel approach, because it allows us to estimate the effect of the prior-adoption variable, which is on the microlevel. We measure prior adoption (PA) by the total number of products actually owned. On average the respondents owned 8.23 products, with a standard deviation of 3.41 .

From the tables we constructed a vector with values of zero and one for all possible combinations of technologies for all respondents; the vector consisted of 5640 observations.

First, to determine which combinations (if any) were perceived as clusters we fitted a binomial random-effects model with an intercept dependent on the respondent, using the lme4 package (Bates \& Sarkar, 2006) of the R-program (R Development Core Team, 2007). As a dependent variable we used the dummy vector with the links made by all respondents; the independent variable was a factor variable, containing all 120 possible combinations.

Next, to test Hypothesis 1, we estimated a binomial random-effects model with an intercept dependent on the respondent. The model predicts the probability of each perceived link by the respondents. The independent variable is a nominal variable capturing the four types of functional linkages in order of increasing infrastructural distance (overlapping function is the reference category).

To estimate the moderating effect of Hypothesis 2, we added interaction terms between the factor that captures functional linkages and the level of prior adoption of consumers (PA). In order to determine the effect of prior adoption for each type of link, we also inserted the prior-adoption variable in four separate models where the dependent variable relates to perceived links based on the four types of links.

To find evidence for our theoretical arguments about perceived clusters, we analyzed the interview question with respect to the motives used by respondents to form their clusters. This was done by interpreting and coding the text fragments of the answers with simple labels for each type of link. This is a way of testing whether our theoretical explanations about the reasons for clustering were correct. The coding was checked for intersubjectivity to ensure a correct interpretation of the text. We thus have four labels, one for each type of link. In analyzing the interviews we found however that the arguments for linking were often a mixture of the labels. In those cases all relevant labels were attached to the text fragment. The number of times a certain label was mentioned is an indicator for the validity of our results (Baarda, De Goede, \& Teunissen, 2005).
Results.

Statistical analysis. In Appendix B the results of the analysis aimed at identifying the clusters perceived by the respondents are presented. To ease interpretation, we show the actual number of times the respondents clustered the technologies together, but the asterisks indicate the $p$ values resulting from the analysis. The significant values indicate that the likelihood that the two technologies are perceived to be linked significantly departs from what is expected on the basis of chance alone. Establishing clusters has a high degree of arbitrariness. We have chosen to look at all links above a threshold value that gives a relatively coherent pattern; in this case this was 21 links. This has no further consequence for the rest of the analysis, which will take into account all links at the individual level. The sole purpose here is to see whether technology clusters can actually be discovered. From Appendix B we can distinguish relatively coherent patterns of clusters if we look at the links that are mentioned more than 21 times. The general result, presented in Table 1, is that there are indeed two main clusters, one around the TV and one around the PC. A third cluster is built around smaller communication devices. A fourth cluster is built around music players. Only the position of the PDA is somewhat ambiguous, because it ends up being in two clusters. These results resemble those in Perse and Courtright (1993) with regard to the TV cluster and the music cluster.

Table 2 presents the estimates of the random effects models that test Hypotheses 1 and 2. "Overlapping functions" is the reference category and has therefore no estimate.

The model predicting the effect of the type of link on the likelihood of linking shows that the larger the distance between the technologies becomes, the smaller the likelihood of linking is. In other words, compared to linking based on overlapping functions, linking on the basis of functional interdependencies has a smaller likelihood, followed by linking based on shared base technologies, and thereafter followed by the unknown type of linking. This confirms Hypothesis 1 and implies two corollary results that are in line with our expectations. First, overlapping functions is the most important factor for consumers to perceive two products as similar. Second, the unknown category is the least important factor for predicting clusters, indicating that considerations not based on functional linkages, such as lifestyle or product attributes

TABLE 1. The clusters that can be found from Appendix B based on 21 perceived links or more.

\begin{tabular}{ll}
\hline Cluster & \multicolumn{1}{c}{ Technologies } \\
\hline 1 & TV, HDTV, FPTV, DVD player, Dolby surround \\
2 & Desktop, laptop, broadband Internet, webcam, game \\
& console, PDA \\
3 & Mobile phone, mobile phone with camera, digital \\
& camera, PDA \\
4 & MP3 player, iPod \\
\hline
\end{tabular}


TABLE 2. The results of the random effect models predicting the likelihood of perceived links.

\begin{tabular}{|c|c|c|c|c|c|c|}
\hline $\mathrm{Y}=$ Prob (Perceived link) & All types & Interaction model & OF & FI & SBT & Unk \\
\hline Intercept & 0.158 & $-0.969^{* *}$ & $-1.020 * *$ & $-1.556^{* * *}$ & $-2.231 * * *$ & $-2.729 * * *$ \\
\hline \multicolumn{7}{|l|}{$\mathrm{OL}$} \\
\hline FI & $-0.673 * * *$ & $-0.601 * *$ & & & & \\
\hline SBT & $-1.384 * * *$ & $-1.243 * * *$ & & & & \\
\hline Unk & $-2.630 * * *$ & $-1.735^{* * *}$ & & & & \\
\hline PA & & $0.137 * * *$ & $0.145^{* * *}$ & $0.124 * * *$ & $0.115^{* *}$ & 0.014 \\
\hline $\mathrm{FI}^{*} \mathrm{PA}$ & & -0.010 & & & & \\
\hline $\mathrm{SBT} * \mathrm{PA}$ & & -0.018 & & & & \\
\hline Unk $*$ PA & & $-0.104 * * *$ & & & & \\
\hline AIC & 5195 & 5180 & 907 & 1719 & 1035 & 1527 \\
\hline Number of respondents & 47 & 47 & 47 & 47 & 47 & 47 \\
\hline Number of observations & 5640 & 5640 & 705 & 1363 & 987 & 2585 \\
\hline
\end{tabular}

$* * * p<0.01, * * p<0.05, * p<0.1$.

like brand, matter the least for perceived clusters in consumer electronics. We will see how the latter result is also confirmed by the findings from the qualitative analysis.

The model that adds the level of prior adoption and its interaction with the type of link (interaction model) shows that there is a moderating effect between the unknown types of links and the level of prior adoption. In the last four columns we explore this interaction further, by using prior adoption as a direct predictor for the probability of linking for each of the four linking categories. This enables us to determine the type of linking to which prior adoption is significantly related. It turns out that prior adoption has a significant positive influence on the likelihood of linking on the basis of overlapping functions, functional interdependencies, and on the same base technology. There is no effect for the unknown types of linking. This indicates that whatever the level of prior adoption, consumers do not differentiate much between types of links when clustering. What we can say is that people with a higher level of prior adoption make more links in general. This partly refutes Hypothesis 2, as individuals with a higher level of prior adoption do perceive clusters more based on functional interdependencies, but nonexperts do not perceive clusters more on the basis of overlapping functions. The partial rejection of Hypothesis 2 implies that in our design we did not succeed in confirming the theories of Gregan-Paxton and John (1997), and neither did we replicate the results of Vishwanath and Chen (2006). There may be several explanations for this. First, our sample size might have been too small; second, the way of relating the technologies may not have sufficiently allowed for detecting different types of linkages other than functional ones; third, the prior adoption of the respondents might not have been differentiated enough to detect any statistically significant differences.

The sample-size limitation is probably not the main issue, considering the fact that we had 120 observations for each respondent. The research design was focused on identifying clusters over all technologies, without any limitations to the size of the cluster. This is a difference with respect to Vishwanath and Chen (2006) who only researched pairs of technologies. Our study did not however instruct respondents on any number of possible linkages, nor on the types of linkages. Probably the respondents' desire for parsimony was stronger than their distinction between different types of links or other possible means of relating technologies. The range in prior adoption was large among the respondents. The set of technologies also contained some very new products next to more conventional products. The respondents were able to categorize these new products in a sensible way, based on the prior adoption they already had of other products.

These considerations lead us to believe that the theoretical arguments elaborated for Hypothesis 2 are still correct, and might be confirmed in the controlled situation of a laboratory experiment such as Vishwanath and Chen (2006) did. However the effects may be too subtle to be confirmed when transferred to a real-life context (Campbell \& Stanley, 1966). If we had added an almost totally unknown product, a really new product or even a nonexistent product to the set, we might have found different effects. This is however far from reality in our particular product domain, where most products are related functionally and therefore familiar to their users.

Qualitative Analysis. The great majority of the text fragments of the respondents explaining their grouping indeed point to clustering based on functionality and infrastructure. Some illustrative examples (all translated from Dutch) follow below. The first one is from a 50-year-old woman who had moderate experience with consumer electronics. She describes her motivation for grouping the desktop, the laptop, broadband Internet, the webcam, and the PDA:

Interviewer: Could you tell me why you have made these groups?

Respondent: Yes, the computer and the laptop are computers of course.

Interviewer: Is that your first group?

Respondent: That is my first group indeed. Broadband Internet also belongs to that group. I wouldn't know were else to put it than with a computer. A webcam is also connected to a computer. Then I also have the personal digital assistant, which is a sort of computerized agenda, I believe. 
This example shows that the respondent started reasoning from overlapping functions (the laptop and the desktop computer) and then added other technologies to the cluster that can be connected the base technology. Another example comes from an inexperienced 76-year-old male, who explains his motivation for clustering the desktop, the laptop, the game console, and the webcam:

Interviewer: Why did you put these items together into one group?

Respondent: This is a kind of computer? [Referring to the notebook]

Interviewer: Yes, it is a kind of computer.

Respondent: At least, I always see my son-in-law walking around with one. Well and this is a game computer. And a webcam, I don't know it, but you always hear that there is trouble with those things, with all of those dirty old men. You also connect those to a computer, don't you? So I thought, yes.

We see the same pattern here. The respondent starts reasoning from overlapping functions (the desktop, the game console, and the laptop) and afterwards (via a step of irrelevant information) he also connects a device that is functionally dependent on a computer.

We have many more examples of this kind of reasoning. Most arguments for clustering contained a mixture of functional overlap and functional interdependencies. There were other sporadic arguments for clustering, like that it appealed to young people or because the items were gadgets. An example that illustrates this was given by a 19-year-old woman:

Interviewer: What can you tell me about this group? [Referring to a group consisting of the iPod, the MP3 player and the digital camera]

Respondent: Yes ... at first I had grouped the things that produce music together, but then I also added the camera. These are all playthings. It is not like you need to own them, but they are fun to have. Well, perhaps the camera can be an item that certain people need, of course. However, for me it is not necessary, but it is a nice toy.

In this excerpt the respondent started reasoning from an overlapping functions perspective, but she ended by concluding that the items in this group were playthings. This is an example of clustering on introspective properties; it describes the respondent's subjective perspective on the object (Yeh \& Barsalou, 2006). Therefore this fragment was coded into the residual "Unknown" category.

In general however the arguments from the interviews confirm our findings in the models. Of the 193 text fragments that were analyzed, 165 referred to a mixture of overlapping functions and functional interdependencies and a shared base technology. Only 28 fragments referred to other arguments.

The results of the qualitative study show that the infrastructural distance between technologies is the most important determinant for linking two technologies. This is in line with the claims of LaRose and Atkin (1992). Alternative explanations like lifestyle are less prominent; clustering starts from functionality. This result is in line with the estimated effects associated with the "Unknown" category in the statistical analysis.

To summarize the findings from Study 1:

- The type of link predicts the likelihood of perceived clustering. The larger the infrastructural distance between two technologies, the smaller the likelihood of perceived linking between technologies becomes.

- Technology clusters are perceived mostly based on functional linkages, while perceived similarities among products based on other considerations are only marginally used.

- The larger the level of prior adoption the larger the likelihood of linking technologies based on overlapping functions, functional interdependencies, and a shared base technology.

Finally, we would like to make a note on methodology. We believe that the motivations behind perceived clustering are better analyzed with qualitative research methods, while evaluating ownership clusters is better done with quantitative research. In our qualitative research we have recovered our theoretical framework in the answers given in the interviews. These answers supported our theory and the theory predicted the clusters correctly. This makes the findings of Study 1 reliable and a valid predictor for Study 2, although we do recognize that the sample size for Study 1 is rather small.

\section{Study 2: A Quantitative Survey About Ownership Clusters in Consumer Electronics}

Methods.

Sample and data collection. A survey was administered by students of an introductory research methodology course to consumers. Respondents were approached to fill in the questionnaires in streets and public places all over the Netherlands. The written questionnaire enquired, among other things, whether the consumers owned the previously mentioned technologies. Since the ordinary TV is owned by $98 \%$ of all households in the Netherlands (CBS, 2007), ${ }^{9}$ it was not included in the questionnaire. It would have too little discriminating value to be useful. Quota by age groups and sex were used to ensure a representative sample. This resulted in a response of 2094 consumers, varying in age between 16 and 88 years of age $($ mean $=44.3) ; 1046$ respondents were male, 1048 were female.

Analysis. All questions regarding ownership of the products were recoded to dummies with value 0 for not owning the product, and value 1 for owning the product. The percentages of ownership are displayed in Table 3. The mean number of products owned was 6.46 with a standard deviation of 2.99. Clearly, there is a wide spread in diffusion among the technologies.

\footnotetext{
${ }^{9}$ This is a choice that is reasonable for the situation in the Netherlands. Studies concerned with countries or periods with lower diffusion rates for the TV should include this product in the analysis.
} 
TABLE 3. The ownership percentages of the 15 technologies.

\begin{tabular}{lccc}
\hline & Valid N & No & Yes \\
\hline PDA & 2084 & $88.7 \%$ & $11.3 \%$ \\
HDTV & 2074 & $88.5 \%$ & $11.5 \%$ \\
iPod & 2079 & $82.5 \%$ & $17.5 \%$ \\
Flat panel TV & 2085 & $80.4 \%$ & $19.6 \%$ \\
Game console & 2087 & $77.1 \%$ & $22.9 \%$ \\
Webcam & 2078 & $68.4 \%$ & $31.6 \%$ \\
MP3 player & 2080 & $66.3 \%$ & $33.8 \%$ \\
Notebook & 2084 & $64.7 \%$ & $35.3 \%$ \\
Dolby surround & 2078 & $61.5 \%$ & $38.5 \%$ \\
Mobile phone with camera & 2078 & $51.9 \%$ & $48.1 \%$ \\
Digital camera & 2084 & $41.0 \%$ & $59.0 \%$ \\
Broadband Internet & 2073 & $26.0 \%$ & $74.0 \%$ \\
Desktop computer & 2083 & $24.8 \%$ & $75.2 \%$ \\
DVD player & 2086 & $21.5 \%$ & $78.5 \%$ \\
Mobile phone & 2084 & $9.3 \%$ & $90.7 \%$ \\
\hline
\end{tabular}

\begin{tabular}{l|l|l|}
\multicolumn{1}{c}{$0\left(\mathrm{C}_{0}\right)$} & \multicolumn{1}{c}{$1\left(\mathrm{C}_{1}\right)$} \\
\cline { 2 - 3 } $0\left(\mathrm{R}_{0}\right)$ & $\mathrm{a}$ & $\mathrm{b}$ \\
\cline { 2 - 3 } $1\left(\mathrm{R}_{1}\right)$ & $\mathrm{c}$ & $\mathrm{d}$ \\
\cline { 2 - 3 } & &
\end{tabular}

FIG. 2. A simple two by two matrix. Cell d represents the ownership links between two technologies.

Since we have no microlevel variables that we want to test, there is no need to build a random-effects model similar to that in the previous study. Instead, we look for an appropriate binary association measure for a simple $2 \times 2$ matrix (Figure 2) to indicate combined ownership. The rows represent Technology 1 and the columns represent Technology 2 . A value of 0 means that the technology is not owned and a value 1 means that the technology is owned. The combined ownership is represented by cell $d$ (the individual owns both items).

Sneath and Sokal (1973) mention various binary association measures like the simple matching coefficient, the Yule coefficient, and the asymmetric Jaccard coefficient. However, due to the large spread in the frequency distributions, none of these measures is applicable. Two widely diffused technologies will automatically have a higher association, because many of the matches in cell $d$ will not be based on chance. If we take, for example, two technologies that are both owned by $90 \%$ of the population, then there is already a guaranteed match in $80 \%$ of the cases. Further, the combined ownership of less widely diffused technologies will be underestimated, because the maximum number of potential matches is lower than with widely diffused technologies. Measures such as the Jaccard coefficient tend to underestimate the low diffused relationships and tend to overestimate the high diffused technologies. Therefore we consider only the number of pairs based on chance. We use the following equation to associate these pairs:

$$
\text { match }=\frac{d-\min d}{\max d-\min d}
$$

where

match $=$ matching coefficient between 0 and 1

$$
d=\text { value of cell } d
$$

$\min d=$ the minimum value of cell $d$

$\max d=$ maximum value of cell $d$

We calculate the association for each possible link between the 15 technologies; this results in 105 different observations. These observations can once again be written as a vector. We test the interaction of Hypothesis 3 with the use of an analysis of covariance (ANCOVA). The dependent variable is the matching coefficient vector for each possible link. The two independent variables are the nominal variable indicating the four types of linking and a variable that represents the perceived links from Study 1 (see Appendix B). Furthermore, we consider an interaction term between the two independent variables. If the interaction term between perceived linkages and overlapping functions is significantly lower than the other interaction terms, then Hypothesis 3 is considered to be confirmed. As control variables we added the diffusion percentages of both technologies. We indicate the most diffused technology as Technology 1, and the other as Technology 2.

\section{Results}

Appendix $\mathrm{C}$ displays the results of the binary-association procedure. The table forms the basis for identifying clusters in ownership. Each cell represents the matching coefficient between the technologies. The larger the matching coefficient, the larger the probability that two technologies are owned in combination with each other. The first thing we notice on the basis of this table is that there is a relatively strong triangular structure within data among the widely diffused technologies. This can be seen because the matching coefficients are higher for the widely diffused technologies than they are for the less widely diffused technologies.

Despite our correction for guaranteed matches, widely diffused technologies are related to most other technologies. This justifies our choice to use diffusion as a control variable in the models. Table 4 displays the results of the ANCOVA models.

The first model is the base model, which only contains the control variables; these variables already explain $83.9 \%$ of the variance. The diffusion of a technology is thus by far the most important factor in predicting ownership clusters, even after controlling for guaranteed matches. The second model predicts ownership clusters based only on the factor capturing the types of functional linkages. Compared to the reference category all types of links appear to be equally strong. The third model includes the perceived links and the control variables. There is a significant effect of perceived links on likelihood of ownership, but this is only a modest improvement in $R$-squared compared to the base model. The fourth model includes an interaction term between the type of link and the perceived links. Overlapping functions in interaction with perceived links leads to a significantly lower chance of combined ownership: Hypothesis 3 is thus confirmed. 
TABLE 4. The results of the ANCOVA models predicting the aggregate amount of ownership linkages.

\begin{tabular}{|c|c|c|c|c|c|c|c|c|}
\hline & $\begin{array}{l}\text { Base } \\
\text { model } \\
(1)\end{array}$ & $\begin{array}{c}\text { Model } \\
\text { categories } \\
\text { (2) }\end{array}$ & $\begin{array}{l}\text { Model } \\
\text { perceived } \\
\text { links } \\
\text { (3) }\end{array}$ & $\begin{array}{l}\text { Interaction } \\
\text { model } \\
(4)\end{array}$ & $\begin{array}{c}\text { Base } \\
\text { model low } \\
\text { diffusion } \\
(5)\end{array}$ & $\begin{array}{c}\text { Base } \\
\text { model high } \\
\text { diffusion } \\
\text { (6) }\end{array}$ & $\begin{array}{c}\text { Model } \\
\text { perceived } \\
\text { links low } \\
\text { diffusion } \\
\quad(7)\end{array}$ & $\begin{array}{c}\text { Model } \\
\text { perceived } \\
\text { links high } \\
\text { diffusion } \\
\text { (8) }\end{array}$ \\
\hline Intercept & .306 & .270 & 0.281 & $.245 * * *$ & $.219 * * *$ & $.454 * * *$ & $.188^{* * *}$ & $.439 * * *$ \\
\hline OL & & $.054 * *$ & & $.109 * *$ & & & & \\
\hline FI & & $.050 * * *$ & & .045 & & & & \\
\hline SBT & & $.058 * * *$ & & $.079 * *$ & & & & \\
\hline Unk (reference category) & & 0 & & 0 & & & & \\
\hline Perceived links (from study 1 ) & & & $.092 * * *$ & $.218^{* * *}$ & & & $.166^{* * *}$ & .049 \\
\hline $\begin{array}{l}\text { Diffusion technology } 1 \\
\text { (most diffused technology) }\end{array}$ & $.009 * * *$ & $.009 * * *$ & $.009 * * *$ & $.009 * * *$ & $.010 * * *$ & $.007 * * *$ & $.009^{* * *}$ & $.007 * * *$ \\
\hline $\begin{array}{l}\text { Diffusion technology } 2 \\
\text { (least diffused technology) }\end{array}$ & $-.005^{* * *}$ & $-.005^{* * *}$ & $-.005^{* * *}$ & $-.005^{* * *}$ & -.003 & $-.006^{* * *}$ & -.003 & $-.006^{* * *}$ \\
\hline Perceived links * OL & & & & $-.272 * *$ & & & & \\
\hline Perceived links * FI & & & & -.148 & & & & \\
\hline Perceived links * SBT & & & & $-.206 *$ & & & & \\
\hline Perceived links * Unk & & & & 0 & & & & \\
\hline$N$ & 105 & 105 & 105 & 105 & 43 & 62 & 43 & 62 \\
\hline Adj. $R^{2}$ & 0.839 & 0.855 & 0.854 & 0.863 & 0.825 & 0.812 & 0.866 & 0.817 \\
\hline
\end{tabular}

*** $p<0.01, * * p<0.05, * p<0.1$.

To get more insight into the role of the diffusion of technologies, we have also split the dataset into two subsets. One subset contains all relationships of the widely diffused technologies, and the other contains the relationships of the less-diffused technologies. To determine this we multiplied the diffusion percentage of Technology 1 with the diffusion percentage of Technology 2 (see Table 3 for the percentages). One subset contains the relationships where the product is $<1000$ (43 cases) and the other subset contains the other relations (62 cases). For both models we estimated again a base model (Models 5 and 6 in the table) and a model with the perceived links variable (Models 7 and 8). Both base models perform well, although the diffusion of Technology 2 does not play a significant role in likelihood of combined ownership for the less-diffused technologies. In Model 7, the perceived links are significant, but not in Model 8. The main finding here is that perceived links from Study 1 only play a significant role for the less-diffused technologies, but not for the more diffused ones.

Study 2 shows first of all that, even after a correction for guaranteed linkages, the rate of diffusion is by far the most important predictor for ownership clusters. There is a relatively strong tendency to buy some technologies first, unrelated to their cluster, and then purchase other technologies that happen to be part of a technology cluster. Base technologies still remain conditional for adopting peripheral technologies, but they explain relative little variance of the patterns in ownership. Perceived links do have a significant interaction with overlapping functions, as was predicted. However, compared to the base model the improvement in fit is negligible (only .024 in adjusted $R$-squared). Perceived links do play a stronger role in predicting the likelihood of combined ownership in case of less-diffused technologies.
Summarized findings of Study 2:

- The diffusion of the technologies is by far the most important factor in predicting ownership clusters.

- Perceived clusters are significantly less predictive for ownership clusters, in case of overlapping functions, compared to other types of links.

- Perceived clusters are significantly predictive for ownership clusters when less-diffused technologies are considered, but not in case of highly diffused technologies.

\section{Conclusions and Discussion}

This paper aimed to contribute to a better understanding of technology clusters in consumer electronics. In this final section we discuss our main findings and their theoretical and practical implications.

As discussed in the introduction, our aim was twofold. First, we aimed to propose a theoretical mechanism that explains the formation of both perceived clusters and clusters in ownership, while at the same time testing whether perceived clusters are predictors of actual ownership patterns.

We have shown that perceived clusters in consumer electronics are significantly determined by functional linkages based on the underlying infrastructure of such products. This result stems both from a qualitative study uncovering motives behind consumers' categorizations and from a quantitative analysis of the effects of different types of product properties. Factors not related to functional linkages, such as lifestyle considerations, are not good predictors of perceived clusters in consumer electronics.

While perceived clusters are primarily based on functionality, ownership clusters are more likely to be based on the diffusion of the technologies. Ownership clustering starts 
from a broad base of technologies that most people own, after which consumers adopt additional parts of one or more clusters according to individual preferences and external circumstances. Starting to adopt a cluster itself may be based on lifestyle, but how the cluster is composed is based on the types of linkages.

The main implication of these results for the literature on technology clusters is that a clear conceptual distinction between perceived and ownership clusters is worthwhile to pursue if one wants to understand the composition of clusters and not take them as exogenous entities.

Our findings also have several implications for diffusion studies in general. First, when predicting the adoption of a certain innovation, many authors include the ownership of other technologies as a predictor in their models (e.g., Leung \& Wie, 1999; Vishwanath \& Goldhaber, 2003). Positive findings from these modelling efforts should not always be attributed to technology clustering. The existence of technology clusters cannot be entirely explained by such results. In fact, the underlying explanations for technology clustering can be rather complex, and they depend on the type of links that connect the technologies. Moreover, positive results can also be the result of a high diffusion rate of one of the products, which will automatically give a large correlation. Future studies should thus be careful in adding ownership variables to their models. The method used to determine the clusters in Study 2 can correct for the high diffusion rate of certain technologies, but even after the correction the diffusion rate has a large influence on the combined ownership of technologies. Our study indicates that for highly diffused technologies, the perceived relationship between technologies is a far less likely explanation for the combined ownership of technologies. This is a consideration that ought to be taken into account in future modelling efforts.

Our second aim in this paper was to investigate the role of prior adoption on technology clusters. We have found that consumers with a higher level of prior adoption perceive clusters based on functional linkages, but without a strong preference for one of the types of linkages considered. Our theoretical prediction was that more expert consumers would use functional interdependences more than overlapping functions. We did not find evidence for this specific claim, but we discussed possible explanations. However, we did find a direct positive relationship between prior adoption and the probability of linking. This implies that experienced consumers are better able to place the innovations into a more detailed context. It exemplifies the fact that in any innovation communication process it is important to tailor the message to the level of a consumer's prior adoption. Based on our results, this is more important than differentiating between types of linkages.

Finally, we wish to conclude by indicating two avenues for further research. On one hand, a further challenge relates to situations in which functional linkages also depend on product attributes. This is likely to be the case when infrastructural linkages differ across brands. Take the example of the choice of a game console cluster. This cluster starts with the purchase of a television, after which almost any type of game console can be bought. Once the choice for a certain brand or type of game console has been made (e.g., Nintendo Wii, Xbox, or Playstation 3), consumers are locked in a certain path. They are bound to the products (video games, controllers, and other extensions) that the specific console has to offer, unless they are willing to invest in another type of console. The prior adoption of consumers also gets more specialized as the cluster gets more specialized. In case of switching, consumers can apply many of their basic skills in the new cluster, but the more specialized knowledge cannot be applied in the new situation.

Further research could also focus on other product domains, to find out how consumers relate products that are not explicitly physically connected to each other. This gives a larger probability of linking technologies based on other aspects of Yeh and Barsalou (2006), next to taxonomic concepts. Also the addition of some less known or even nonexistent technologies to a set of technologies could provide interesting results in a future study. In a related vein, research could aim at a richer conceptualization of technology clusters by taking into account more attribute levels. Diversified product attributes may render a certain product more attractive than another even when the two are functional substitutes.

\section{Acknowledgments}

We would like to thank Jaco van Dijk for helping to collect and analyze the qualitative data, Rogier Donders for his help in carrying out the research, and Martin Dijst and Jenny van Doorn for helpful comments. Finally, we thank the two anonymous reviewers for their helpful comments.

\section{References}

Baarda, D.B., De Goede, M.P.M., \& Teunissen, J. (2005). Basisboek kwalitatief onderzoek. [The basics of qualitative research]. Houten, Netherlands: Stenfert Kroese.

Bates, D., \& Sarkar, D. (2006). Ime4: Linear mixed-effects models using S4 classes [Computer software].

Bussele, R., Reagan, J., Pinkleton, B., \& Jackson, K. (1999). Factors affecting Internet ese in a saturated-access population. Telematics and Informatics, $16,45-99$.

Campbell, D.T., \& Stanley, J.C. (1966). Experimental and quasiexperimental designs for research. London: Houghton Mifflin.

CBS. (2007). Statline database. Heerlen: Centraal Bureau voor de Statistiek.

Cowles, D. (1989). Consumer perceptions of interactive media. Journal of Broadcasting \& Electronic Media, 33, 83-89.

Ettema, J.S. (1984). Three phases in the creation of information inequities: An empirical assessment of a prototype Videotex system. Journal of Broadcasting, 28, 383-395.

Gregan-Paxton, J., \& John, D.R. (1997). Consumer learning by analogy: A model of internal knowledge transfer. Journal of Consumer Research, $24,266-284$.

Kwortnik, R.J., \& Ross, W.T. (2007). The role of positive emotions in experiential decisions. International Journal of Research in Marketing, 24, 324-335.

LaRose, R., \& Atkin, D. (1992). Audiotext and the Reinvention of the Telephone as a Mass Medium. Journalism Quarterly, 69(2), 413-421. 
LaRose, R., \& Hoag, A. (1996). Organizational adoptions of the internet and the clustering of innovations. Telematics and Informatics, 13, 49-61.

Leung, L., \& Wei, R. (1999). Who are the mobile phone have-nots? New Media \& Society, 1, 209-226.

Lin, C.A. (1998). Exploring personal computer adoption dynamics. Journal of Broadcasting \& Electronic Media, 42, 95-112.

Moreau, C.P., Markman, A.B., \& Lehmann, D.R. (2001). "What is it?" Categorization flexibility and consumers' responses to really new products. Journal of Consumer Research, 27, 489-498.

Nedungadi, P., Chattopadhyay, A., \& Muthukrishnan, A.V. (2001). Category structure, brand recall, and choice. International Journal of Research in Marketing, 18, 191-202.

Neuendorf, K.A., Atkin, D., \& Jeffres, L.W. (1998). Understanding adopters of audio information innovations. Journal of Broadcasting \& Electronic Media, 42, 80-93.

Perse, E.M., \& Courtright, J.A. (1993). Normative images of communication media: Mass and interpersonal channels in the new media environment. Human Communication Research, 19, 485-503.

R Development Core Team. (2007). R: A language and environment for statistical computing [Computer Software]. Vienna, Austria: R Foundation for Statistical Computing.
Rogers, E.M. (2003). Diffusion of innovations (5th ed.). New York: Free Press.

Rosa, J.A., \& Porac, J.F. (2002). Categorization bases and their influence on product category knowledge structures. Psychology \& Marketing, 19, 503-531.

Sneath, P.H.A., \& Sokal, R.R. (1973). Numerical taxonomy. San Fransico: W. H. Freeman and Company.

Snijders, T.A.B., \& Bosker, R.J. (1999). Multilevel analysis. London: Sage.

Vishwanath, A., \& Chen, H. (2006). Technology clusters: Using multidimensional scaling to evaluate and structure technology clusters. Journal of the American Society for Information Science and Technology, 57, 1451-1460.

Vishwanath, A., \& Goldhaber, G.M. (2003). An examination of the factors contributing to adoption decisions among late-diffused technology products. New Media \& Society, 5, 547-572.

Warlop, L., Ratneshwar, S., \& van Osselaer, S.M.J. (2005). Distinctive brand cues and memory for product consumption experiences. International Journal of Research in Marketing, 22, 27-44.

Yeh, W.C., \& Barsalou, L.W. (2006). The situated nature of concepts. American Journal of Psychology, 119, 349-384.

\section{Appendix A: The typology of all possible links. OF = Overlapping functions, $\mathrm{FI}=\mathrm{Functional}$ Interdependencies, SBT = Shared base technologies, Unk = Unknown, no relationship.}

\begin{tabular}{|c|c|c|c|c|c|c|c|c|c|c|c|c|c|c|c|}
\hline & Pda & HDTV & Ipod & FPTV & Game & Webcam & Mp3 & $\mathrm{NB}$ & Dolby & MPC & Dicam & Broadint & Desk & DvD & MP \\
\hline \multicolumn{16}{|l|}{ Pda } \\
\hline HDTV & Unk & & & & & & & & & & & & & & \\
\hline Ipod & SBT & Unk & & & & & & & & & & & & & \\
\hline FPTV & Unk & $\mathrm{OF}$ & Unk & & & & & & & & & & & & \\
\hline Game & Unk & FI & Unk & FI & & & & & & & & & & & \\
\hline Webcam & SBT & Unk & SBT & Unk & Unk & & & & & & & & & & \\
\hline Mp3 & OF & Unk & OF & Unk & Unk & SBT & & & & & & & & & \\
\hline NB & FI & Unk & FI & Unk & OF & FI & FI & & & & & & & & \\
\hline Dolby & Unk & FI & SBT & FI & FI & SBT & SBT & FI & & & & & & & \\
\hline MPC & $\mathrm{OF}$ & Unk & OF & Unk & Unk & Unk & OF & FI & Unk & & & & & & \\
\hline Digicam & SBT & FI & SBT & SBT & SBT & SBT & SBT & FI & SBT & OF & & & & & \\
\hline Broadint & SBT & Unk & SBT & Unk & FI & SBT & SBT & FI & SBT & Unk & SBT & & & & \\
\hline Desk & FI & Unk & FI & Unk & OF & FI & FI & OF & FI & Unk & FI & FI & & & \\
\hline Dvd & Unk & FI & Unk & FI & SBT & Unk & Unk & Unk & FI & Unk & Unk & Unk & Unk & & \\
\hline MP & OF & Unk & OF & Unk & Unk & Unk & OF & Unk & Unk & OF & Unk & Unk & Unk & Unk & \\
\hline TV & Unk & $\mathrm{OF}$ & Unk & $\mathrm{OF}$ & FI & Unk & Unk & Unk & FI & Unk & FI & Unk & Unk & FI & Unk \\
\hline
\end{tabular}

Note: The numbers represent the number of times that the connection was made. The asterisks represent the p-value of the binomial random effects model: ***: $p<0.01, * * p<0.05, * p<0.1$. The smaller the $p$-value, the larger the likelihood that the perceived links are not based on chance.

Appendix B: The results of binomial logistic random effects model. The numbers represent the number of times that the connection was made.

\begin{tabular}{|c|c|c|c|c|c|c|c|c|c|c|c|c|c|c|c|}
\hline & Pda & HDTV & Ipod & FPTV & Game & Webcam & Mp3 & NB & Dolby & MPC & Dicam & Broadint & Desk & DvD & MP \\
\hline \multicolumn{16}{|l|}{ Pda } \\
\hline HDTV & 2 & & & & & & & & & & & & & & \\
\hline Ipod & $16^{* *}$ & 4 & & & & & & & & & & & & & \\
\hline FPTV & 1 & $40 * * *$ & 1 & & & & & & & & & & & & \\
\hline Game & $15^{* *}$ & $9^{*}$ & 9 & $12 * *$ & & & & & & & & & & & \\
\hline Webcam & $22 * * *$ & 6 & 8 & 5 & $29 * * *$ & & & & & & & & & & \\
\hline Mp3 & $12^{* *}$ & 1 & $42 * * *$ & 1 & 7 & 6 & & & & & & & & & \\
\hline NB & $27 * * *$ & 2 & $10 *$ & 3 & $27 * * *$ & $39 * * *$ & 9 & & & & & & & & \\
\hline Dolby & 4 & $21 * * *$ & $15^{* *}$ & $25 * * *$ & $10 *$ & $10^{*}$ & $19 * * *$ & 8 & & & & & & & \\
\hline MPC & $25 * * *$ & 2 & $12 *$ & 2 & 3 & 6 & $11^{*}$ & 8 & 2 & & & & & & \\
\hline Digicam & $19 * * *$ & 4 & $15^{* *}$ & 6 & 6 & 9 & $13 *$ & 9 & 5 & $30 * * *$ & & & & & \\
\hline Broadint & $20 * * *$ & 5 & 8 & 6 & $31 * * *$ & $43 * * *$ & 6 & $37 * * *$ & $12 *$ & 5 & 8 & & & & \\
\hline Desk & $20 * * *$ & 4 & 7 & 7 & $32 * * *$ & $42 * * *$ & 6 & $40 * * *$ & $13^{* *}$ & 3 & 7 & $42 * * *$ & & & \\
\hline Dvd & 0 & $35^{* * *}$ & 6 & $39 * * *$ & $11^{*}$ & 5 & 7 & 0 & $29 * * *$ & 3 & 4 & 4 & 3 & & \\
\hline MP & $25 * * *$ & 2 & $13 *$ & 1 & 1 & 4 & $12 *$ & 6 & 5 & $45^{* * *}$ & $22 * * *$ & 3 & 3 & 2 & \\
\hline TV & 1 & $37 * * *$ & 2 & $44 * * *$ & $10^{*}$ & 6 & 1 & 2 & $23 * * *$ & 1 & 4 & 5 & 5 & $40 * * *$ & 2 \\
\hline
\end{tabular}


Appendix C: The results of the binary association procedure.

\begin{tabular}{|c|c|c|c|c|c|c|c|c|c|c|c|c|c|c|}
\hline & Pda & HDTV & Ipod & FPTV & Game & Webcam & Mp3 & NB & Dolby & MPC & Digicam & Broadint & Desk & DvD \\
\hline \multicolumn{15}{|l|}{ Pda } \\
\hline HDTV & 0.22 & & & & & & & & & & & & & \\
\hline Ipod & 0.30 & 0.27 & & & & & & & & & & & & \\
\hline FPTV & 0.34 & 0.62 & 0.29 & & & & & & & & & & & \\
\hline Game & 0.38 & 0.32 & 0.39 & 0.30 & & & & & & & & & & \\
\hline Webcam & 0.53 & 0.46 & 0.53 & 0.42 & 0.57 & & & & & & & & & \\
\hline Mp3 & 0.50 & 0.43 & 0.42 & 0.40 & 0.51 & 0.51 & & & & & & & & \\
\hline NB & 0.71 & 0.50 & 0.52 & 0.46 & 0.48 & 0.47 & 0.46 & & & & & & & \\
\hline Dolby & 0.63 & 0.69 & 0.50 & 0.62 & 0.52 & 0.50 & 0.47 & 0.45 & & & & & & \\
\hline MPC & 0.69 & 0.60 & 0.80 & 0.57 & 0.73 & 0.69 & 0.68 & 0.63 & 0.60 & & & & & \\
\hline Digicam & 0.80 & 0.74 & 0.64 & 0.79 & 0.66 & 0.71 & 0.70 & 0.72 & 0.72 & 0.60 & & & & \\
\hline Broadint & 0.90 & 0.86 & 0.89 & 0.84 & 0.89 & 0.90 & 0.86 & 0.81 & 0.79 & 0.77 & 0.65 & & & \\
\hline Desk & 0.88 & 0.85 & 0.78 & 0.85 & 0.84 & 0.83 & 0.78 & 0.58 & 0.76 & 0.61 & 0.65 & 0.56 & & \\
\hline Dvd & 0.90 & 0.89 & 0.84 & 0.92 & 0.87 & 0.78 & 0.78 & 0.75 & 0.87 & 0.68 & 0.68 & 0.51 & 0.47 & \\
\hline MP & 0.99 & 0.94 & 0.98 & 0.90 & 0.96 & 0.95 & 0.92 & 0.93 & 0.86 & 0.97 & 0.80 & 0.71 & 0.62 & 0.51 \\
\hline
\end{tabular}

